\title{
AC-DC Calibrations With a Pulse-driven AC Josephson Voltage Standard Operated in a Small Cryostat
}

\author{
Helko E. van den Brom, Senior Member, IEEE, Oliver F.O. Kieler, Stephan Bauer, and Ernest \\ Houtzager, Senior Member, IEEE
}

\begin{abstract}
AC-DC measurements have been performed on a commercial thermal transfer standard (TTS) using a pulsedriven AC Josephson voltage standard with a small cryostat enabling shorter voltage leads. A $20 \mathrm{mV}$ RMS output voltage was chosen, close to the maximum value that can be obtained without low-frequency compensation (at a sigma-delta code amplitude of about $10 \%$ ) and close to the upper limit of the TTS in its $22 \mathrm{mV}$ range. The measured deviation at higher frequencies, which is due to the length of the voltage leads, was an order of magnitude smaller than was observed before with a normal cryostat. The observed square dependence on the signal frequency and cable length follows exactly the expected behavior. All results agree well with the calibrations performed using a micropot reference system. Furthermore, the influence of other cables and equipment was investigated. The measurement results can be quantitatively understood both in terms of a lumped circuit description and in terms of reflected waves.
\end{abstract}

Index Terms - AC-DC difference, Josephson voltage standard, measurement standards, measurement techniques, voltage measurement

\section{INTRODUCTION}

$\mathrm{T}$ HE development of a pulse-driven AC Josephson voltage standard (ACJVS) is already going on for 20 years [1] The focus of the developments has been on increasing the output voltage to practical levels without compromising too much on sufficiently large operating margins, i.e., the ranges over which the different bias parameters can be varied without changing the spectral purity of the output signal [2]. The output voltage can be increased to values on the order of $1 \mathrm{~V}$ by increasing the number of junctions in a series array and connecting a number of such arrays, each with its own bias electronics, in series $[3,4]$.

Apart from reaching practical voltage levels, the problem of

Manuscript received July 15, 2016. This work is carried out with funding by the European Union within the EMRP JRP SIB59 Q-WAVE. The EMRP is jointly funded by the EMRP participating countries within EURAMET and the European Union.

H.E. van den Brom and E. Houtzager are with VSL, Dutch Metrology Institute, P.O. Box 654, 2600AR Delft, The Netherlands (email: hvdbrom@vsl.nl).

O.F.O. Kieler and S. Bauer are with Physikalisch-Technische Bundesanstalt (PTB), Bundesallee 100, D-38116 Braunschweig, Germany (email: okieler@ptb.nl). reaching low uncertainties has been addressed as well. At frequencies up to $1 \mathrm{kHz}$, impressive uncertainties have been obtained already by comparisons between the pulse-driven ACJVS and a programmable Josephson voltage standard (PJVS) [5,6] and between two ACJVS systems [7,8]. At higher frequencies it is still very difficult to perform precision measurements with uncertainties lower than obtained when using conventional AC-DC thermal converters [7,9-11]. The major problem is that for high-accuracy AC-DC calibrations the device under test (DUT) and the reference converter are usually connected as close as possible to each other, such that the reference plane of the measurement is close to the input connector of both DUT and reference. In the case of a pulsedriven ACJVS, the calculable AC voltage signals are generated at low temperatures (in our case $4.2 \mathrm{~K}$ ), whereas they are measured with equipment under test at room temperature. The voltage leads and connection cables cause the output voltage to show deviations that scale with frequency squared; deviations of almost $1 \%$ at $1 \mathrm{MHz}$ have been reported for a cryostat with voltage leads of about $1.5 \mathrm{~m}$ [11]. For practical applications, this limits the ACJVS output frequency to approximately $100 \mathrm{kHz}[7,10,11]$.

Recent investigations revealed that the frequency dependence can be explained by reflection of waves at the high impedance of the load [12]. Calculations showed that at first order approximation the voltage at the input of the device under test is increased by a factor that scales with the cable length squared, the signal frequency squared, and the inverse propagation speed of electromagnetic waves through the cable squared, independent of other parameters. This calculation is based on the assumption that the input impedance of the DUT is high and the output impedance of the ACJVS is low compared to the characteristic impedance of the cable. The only fitting parameters are an offset length representing the apparent signal path in the DUT and the propagation speed of the waves in the cable. It was suggested that using a small cryostat would result in much smaller deviations than observed in other work $[10,11]$.

In Ref. [13] we presented measurements obtained using a He 4 cryostat at PTB with a short cryoprobe, which enables a distance of only $70 \mathrm{~cm}$ between the Josephson array and the output connector at room temperature. We connected a commercial Fluke 792A AC-DC thermal transfer standard 


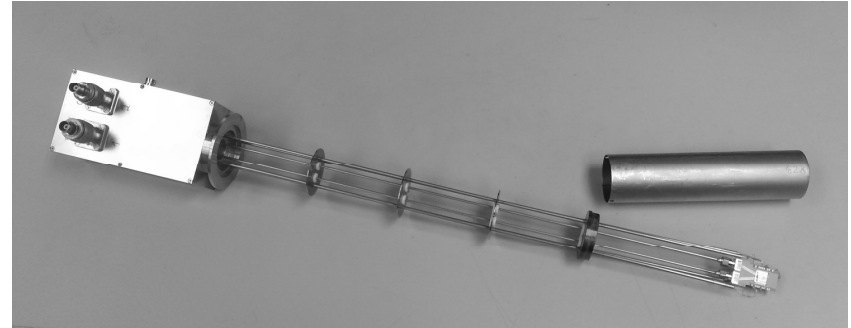

Fig. 1. Photograph of the short cryoprobe with the ACJVS chip connected. the cryoperm shield is visible too. One of the arrays on the chip is connected through a coaxial cable, the other by means of a twisted pair cable. The overall length of the cryoprobe is about $60 \mathrm{~cm}$.

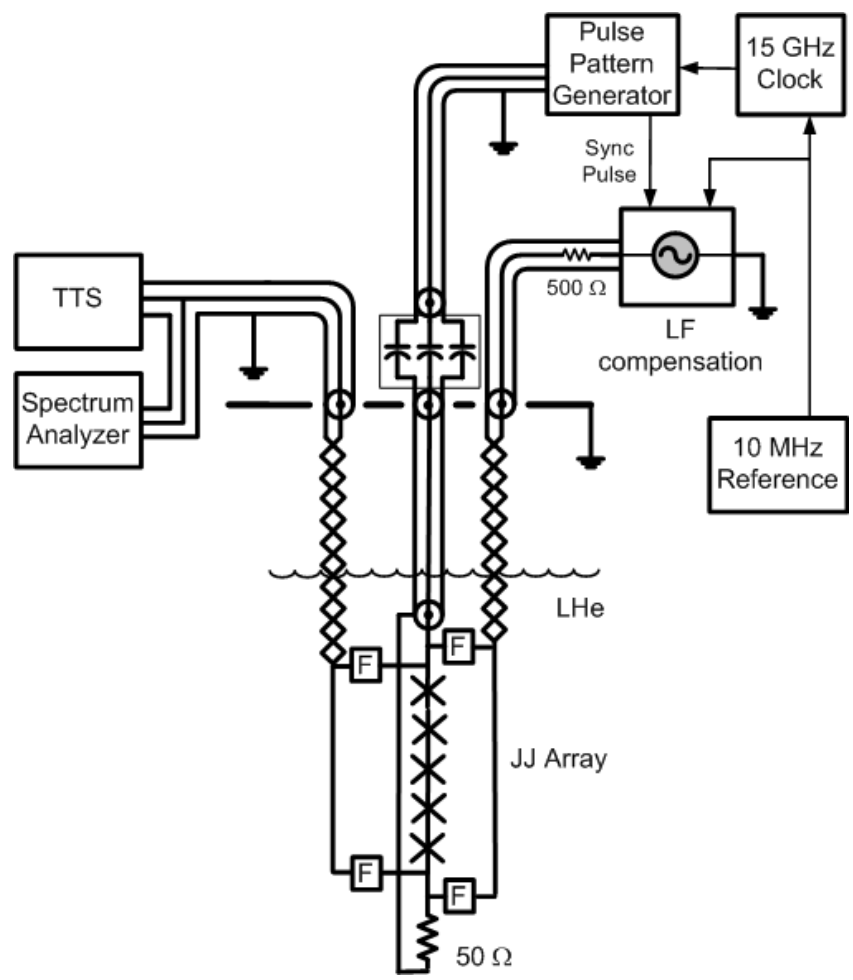

Fig. 2. Schematic overview of the setup. For precision measurements the spectrum analyzer and waveform generator for LF compensation current are disconnected when not needed. The TTS under test is battery operated, with its chassis, guard and Lo terminal to the mains earth connection.

(TTS) for the high accuracy measurements, similar to earlier measurements [7,9-11]. In this paper, we extend on these measurements and provide more details of the experimental setup. Furthermore, measurement results were compared to new calibration values of the TTS obtained by using micropots, resulting in slightly different numbers for the observed uncorrected deviations without changing the general conclusions.

\section{MEASUREMENT APPROACH}

\section{A. Measurement setup}

In the PTB pulse-driven ACJVS [14] a chip with two arrays of 9000 junctions each was installed for the measurements described here. Triple-stacked SNS Josephson junctions (S: superconductor, $\mathrm{N}$ : normal metal) with a normal metal $\mathrm{Nb}_{0.2} \mathrm{Si}_{0.8}$ were used. The critical current of the ACJVS arrays was about $2.7 \mathrm{~mA}$. The arrays showed typical current operation margins of about $1.8 \mathrm{~mA}$ at $20 \mathrm{mV}$ output voltage and about $1.1 \mathrm{~mA}$ at $50 \mathrm{mV}$ output voltage. Instead of operating the ACJVS in the usual cryoprobe that can be inserted in a liquid helium dewar, a new short cryoprobe has been designed and fabricated at PTB for operation in a small He-4 cryostat (see Fig. 1). The Oxford Instruments minicryostat provides a vacuum and liquid nitrogen shield for the liquid helium dewar. Continuous measurements without refilling liquid helium are possible for several hours sufficient to perform all measurements described. In this cryostat, the ACJVS chip is cooled by exchange gas only. This is more than enough to cool the ACJVS chip, because the operation by pulses is not introducing too much microwave power as compared to the PJVS. The distance between the Josephson chip and the N-connector on the head of the cryoprobe at room temperature was only $70 \mathrm{~cm}$, which is a factor of 2 smaller than for the original cryoprobe.

Fig. 2 shows a schematic overview of the measurement setup. A Sympuls BMG-30G pulse-pattern generator delivering positive and negative pulses at a clock frequency of $15 \mathrm{GHz}$ was used [15]. Switching between different signals could be done very fast by using up to 4 different memory benches of the pulse-pattern generator, such that settling times for AC-DC difference measurements are significantly reduced.

Measurements have been performed using a coaxial cable (with inner wire and outer shield both made of silver plated copper, and $50 \Omega$ characteristic impedance) as well as an unshielded twisted pair cable (with unknown characteristic impedance) as voltage leads inside the cryoprobe. The coaxial cable and its soldering connections caused a significant thermal voltage of about $200 \mu \mathrm{V}$, which was compensated for by adding a DC offset in the digital code sent to the pulse pattern generator. The twisted pair cable caused a thermal voltage of less than $20 \mu \mathrm{V}$.

In order to avoid possible ground loops and other disturbances, the low-frequency (LF) compensation circuit was disconnected and the ACJVS was driven with AC coupled bipolar pulses only when generating a RMS voltage of $20 \mathrm{mV}$ [16]. For the generation of $50 \mathrm{mV}$ waveforms we applied the LF compensation as usual $[14,16]$, with one exception: for the waveforms above $500 \mathrm{kHz}$ we used a $500 \Omega$ series resistor (as shown in Fig. 2) to convert the voltage into a current instead of the battery-operated home-built compensation electronics, because of its limited bandwidth. The compensation circuit is basically a differential amplifier followed by conversion from voltage into a current, such that it is isolated from the function generator and to convert it into a current. The operation of the compensation circuit itself has no direct influence on the accuracy of this measurement [9-11]. We obtained stable operation margins for the positive and negative pulse amplitudes and the compensation amplitude and phase, ensuring a quantized voltage output at all times.

The ACJVS output voltage was connected directly to the TTS under investigation, see Fig. 3. The chassis and the guard of the TTS output were connected to ground, and so was the Lo of the ACJVS output (and consequently the TTS input). A 


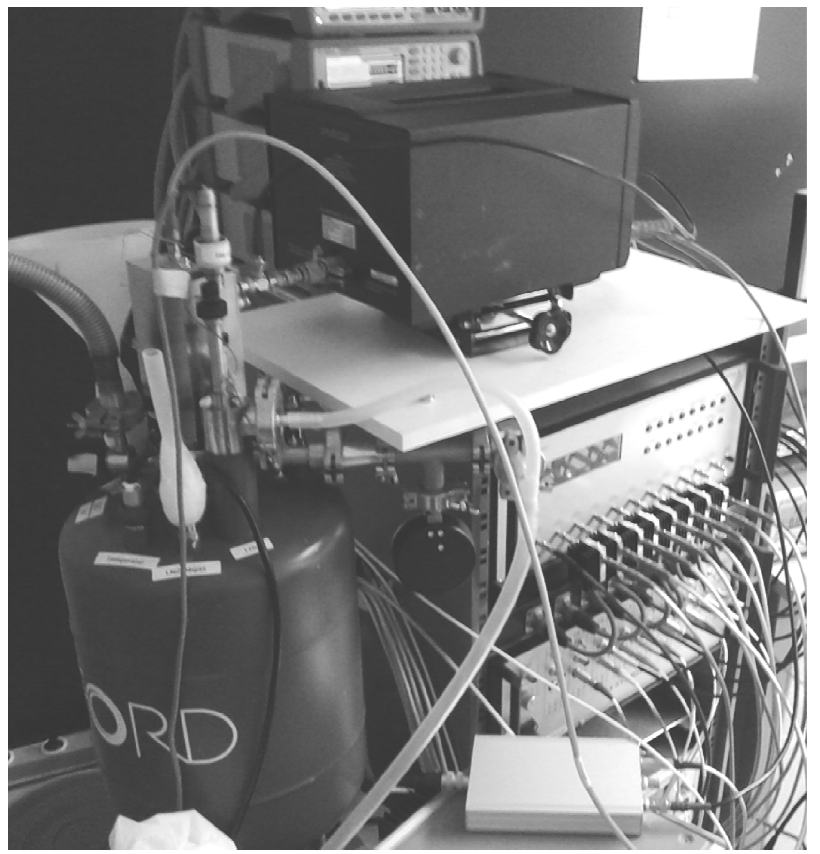

Fig. 3. Photograph of the measurement setup. The short cryostat with the cryoprobe can be seen on the left, the TTS is at the top, and the pulse-pattern generator and home-built LF compensation amplifier box with their 8 outputs at the right. Between the probe head and the TTS one can see a bias tee with a cable connected to the spectrum analyzer (not shown).

tee-connector was used to connect a NI 5922 digitizer to verify the spectral purity of the generated waveform. Both the Lo terminal of the TTS input and the guard and chassis at the output terminals were grounded. A $20 \mathrm{mV}$ output voltage was chosen, close to the maximum value that can be obtained without LF compensation (at a sigma-delta code amplitude of about $10.1 \%$ ) and close to the upper limit of the TTS in its $22 \mathrm{mV}$ range. Note that the TTS in this setting has $10 \mathrm{M} \Omega$ input impedance, whereas the digitizer input impedance is $1 \mathrm{M} \Omega$ with $40 \mathrm{pF}$ in parallel.

\section{B. AC-DC measurements}

AC-DC difference measurements on the TTS were performed as follows. First the AC-DC difference of the TTS at $1 \mathrm{kHz}$ was measured by successively applying a positive DC signal, an AC signal and a negative DC signal as generated by the ACJVS. Then less noise-sensitive AC-AC differences were measured, in which measurements at higher frequencies were compared to the $1 \mathrm{kHz}$ result.

In the experiments presented here, before starting the actual data acquisition, waiting times of 10 seconds after switching between two AC signals turned out to be more than sufficient. For switching between AC and DC signals, however, waiting times of 45 seconds turned out to be necessary.

\section{EXPERIMENTAL RESULTS AND DISCUSSION}

\section{A. Spectra}

For the thermal transfer measurements we synthesized sine waveforms for different frequencies from $1 \mathrm{kHz}$ up to $1 \mathrm{MHz}$ for two different RMS output voltages, i.e., $20 \mathrm{mV}$ and $50 \mathrm{mV}$. The $20 \mathrm{mV}$ waveforms were generated without

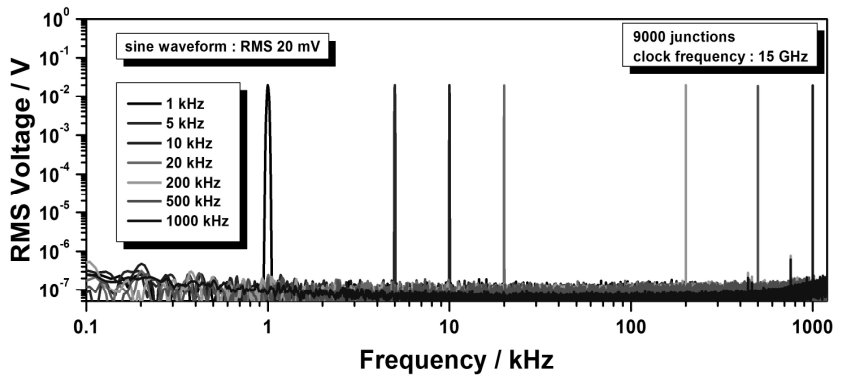

Fig. 4. Frequency spectra of seven sine waves with different signal frequencies and an RMS amplitude of $20 \mathrm{mV}$ synthesized with an ACJVS chip with 9000 Josephson junctions at a clock frequency of $15 \mathrm{GHz}$.

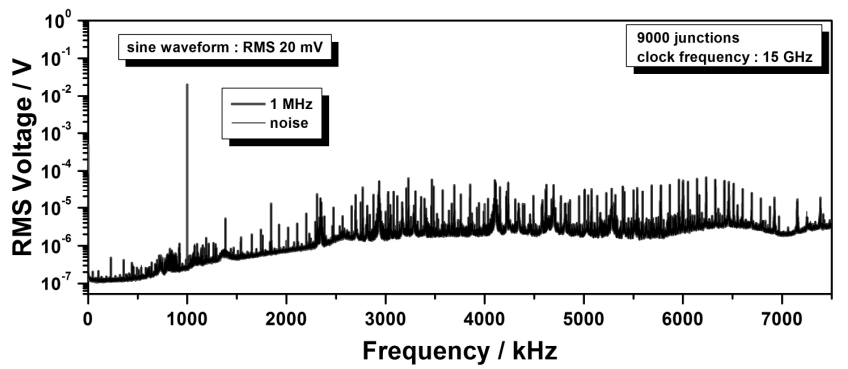

Fig. 5. Frequency spectrum of a $20 \mathrm{mV}, 1 \mathrm{MHz}$ sine wave synthesized with an ACJVS chip with 9000 Josephson junctions at a clock frequency of $15 \mathrm{GHz}$. The noise floor of the digitizer, measured separately using the same settings, is plotted as well.

compensation signal and for the $50 \mathrm{mV}$ waveforms the compensation was applied.

Fig. 4 shows the high-quality frequency spectra for the $20 \mathrm{mV}$ waveforms for seven different signal frequencies obtained without compensation. The noise floor was slightly reduced for the $1 \mathrm{MHz}$ sine wave, because the RMS averaging (10 averages) was switched on during the spectrum measurement with the digitizer. The frequency spectrum for the $1 \mathrm{MHz}$ sine wave is shown in Fig. 5 again, but with extended frequency range. The lower signal-to-noise ratio for these measurements is fully caused by the noise floor of the digitizer which was measured separately and plotted in the same figure as well.

The corresponding frequency spectra for different signal frequencies at $50 \mathrm{mV}$ with compensation showed similar results. However, some minor non-harmonic noise content was observed close to $1 \mathrm{MHz}$, probably due to the resistor used for the compensation signal.

\section{B. AC-DC measurements with direct connection}

In order to get the lowest possible influence of the voltage leads, the TTS was connected directly to the cryoprobe head, with only the tee connector and BNC-to-N connectors in between (see Fig. 3). To avoid possible influence from the digitizer, after tuning the ACJVS the digitizer was disconnected from the setup and the tee connector was covered with an open cap. AC-DC difference values were measured using $20 \mathrm{mV}$ ACJVS signals generated using the first array on the chip, which is connected by means of the coaxial cable inside the cryoprobe, without using LF compensation. For all AC-DC measurements the standard 


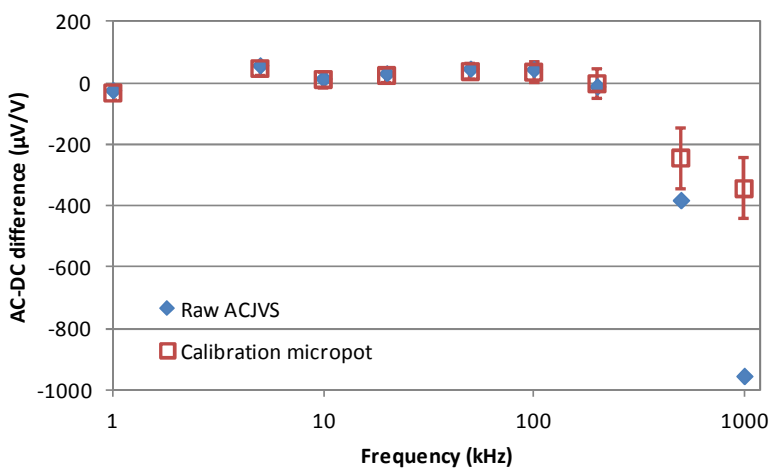

Fig. 6. ACJVS results for the AC-DC difference of the TTS at $20 \mathrm{mV}$ as compared to the PTB micropot calibration results. The measurement data have not been corrected for the influence of the voltage leads. As can be seen, the maximum deviation from the micropot calibration result of the TTS is about $610 \mu \mathrm{V} / \mathrm{V}$ at $1 \mathrm{MHz}$.

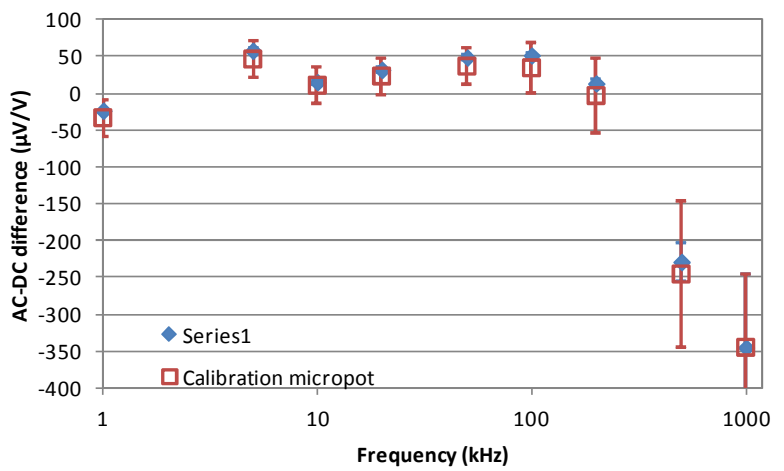

Fig. 7. ACJVS results of the AC-DC difference of the TTS at $20 \mathrm{mV}$, corrected for the squared frequency dependence of the voltage leads. Note that due to the compensation method by definition the $1 \mathrm{MHz}$ value of the corrected data and the micropot calibration result are identical.

deviation was less than $1 \mu \mathrm{V} / \mathrm{V}$.

The AC-DC measurement results are shown in Fig. 6, in which it can be seen that the maximum deviation from the micropot calibration result of the TTS is $610 \mu \mathrm{V} / \mathrm{V}$ at $1 \mathrm{MHz}$. Note that this is more than a factor of 10 less than the almost $1 \%$ deviation observed before [10]. A square frequency dependence of the deviation was observed as expected, although it should be noted that the uncertainty of $100 \mu \mathrm{V} / \mathrm{V}$ of the micropot calibration result becomes a significant factor as compared to the observed difference values.

The measurements at lower frequencies were corrected based on the PTB micropot calibration result at $1 \mathrm{MHz}$, assuming squared frequency dependence, following the procedure explained in Ref. [11]. Fig. 7 shows that the ACJVS results found this way agree very well with the micropot results. The uncertainty bars shown in this figure are a combination of the scaled error of the micropot calibration at $1 \mathrm{MHz}$ and other specific error sources of the ACJVS as explained in Refs. [7,11]. Note that by definition of our compensation method the micropot results and the corrected ACJVS results at $1 \mathrm{MHz}$ are identical.

\section{Second array and other voltage leads}

As a verification of the measurements we performed the

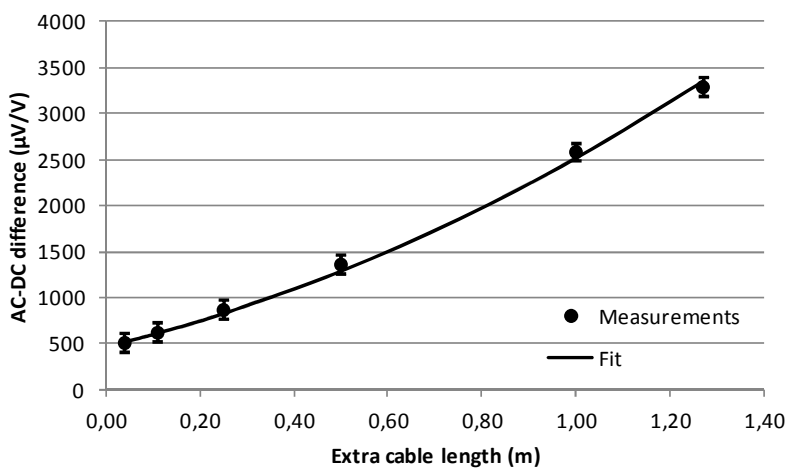

Fig. 8. Difference between the AC-DC measurement results obtained with the ACJVS and the micropot calibration results of the TTS at $20 \mathrm{mV}$ and $1 \mathrm{MHz}$ as a function of the length of the extra cable inserted. The dots represent the measurements, whereas the line is a fitted curve assuming a squared frequency dependence and an offset path length of $75 \mathrm{~cm}$. The uncertainty bars on the measurement values represent the TTS micropot calibration uncertainty.

same type of AC-DC measurements at $20 \mathrm{mV}$ using the second array on the same chip, which was connected to the cryoprobe head using twisted pair cables instead of a coaxial cable. The observed spectrum was identical to that shown in Fig. 4, from which we conclude that the array itself behaves as expected. The standard deviation of the AC-DC measurements was again less than $1 \mu \mathrm{V} / \mathrm{V}$.

The difference between the AC-DC measurement results obtained for the two different cables was only significant for the highest two frequencies of $500 \mathrm{kHz}$ and $1 \mathrm{MHz}$. For frequencies below $100 \mathrm{kHz}$ the difference was within $1 \mu \mathrm{V} / \mathrm{V}$, whereas at the highest frequency of $1 \mathrm{MHz}$ the difference was about $100 \mu \mathrm{V} / \mathrm{V}$. Since the only difference between the two measurements is the voltage leads inside the probe, the observed difference in measurement results can be attributed to a slightly different length of the cable inside the probe or to slightly different cable characteristics.

\section{Cable influence}

As a next experiment, we investigated the dependence of the observed deviation between ACJVS results and micropot calibration results on the length of the voltage leads. Starting from the configuration described in the previous section, we changed the configuration of the system. An extra RG213 coax cable was inserted between the cryoprobe and the TTS. Cables of $25 \mathrm{~cm}, 50 \mathrm{~cm}, 100 \mathrm{~cm}$ and $127 \mathrm{~cm}$ were used, replacing the adapter including the tee-connector with cap which is approximately $4 \mathrm{~cm}$ long. Also an extra male-tofemale adapter was used, resulting in $11 \mathrm{~cm}$ between probe and TTS. As can be seen in Fig. 8, for the largest cable of $127 \mathrm{~cm}$ we found a deviation of $0.33 \%$ at $1 \mathrm{MHz}$. For the direct connection without tee connector, the deviation was only $510 \mu \mathrm{V} / \mathrm{V}$. Note that this is about $100 \mu \mathrm{V} / \mathrm{V}$ lower than observed in the previous sections, where the tee connector was still inserted for practical reasons.

We fitted the observed behavior to squared length dependence with an offset representing both the length of the voltage lead inside the cryoprobe and the path length inside the TTS as a fitting parameter. That is, we fitted the data to a 
curve $y=a \cdot\left(x+x_{0}\right)^{2}$, where $y$ represents the measured deviation, $x$ the length of the cable and $x_{0}$ the offset, and $a$ is a constant of proportionality. In the fitting procedure, the constant of proportionality $a$ was calculated for all measurements presented in Fig. 8 assuming a certain fixed value of $x_{0}$; the standard deviation between the different values of $a$ obtained this way was then minimized by varying $x_{0}$. When assuming the total path length within the cryoprobe and within the TTS to be $x_{0}=75 \mathrm{~cm}$, the fit seems to agree with the data well within the uncertainty limits of the TTS micropot calibration. Hence, we can conclude that the deviation indeed scales nicely with the square of the cable length, as expected.

\section{E. Quantitative explanation of the measurement results}

The observed deviation and the square frequency dependence can be understood both from a lumped circuit approach or from a transmission line approach. When treating the voltage leads as a symmetric T-type segment two inductors with inductance $1 / 2 L$ each, and one capacitor with capacitance $C$ in between, one can see that the voltage $V_{\text {out }}$ at the output of the cable relative to the voltage at the input $V_{i n}=V_{J o s}$ is equal to

$$
V_{\text {out }} / V_{\text {in }}=1 /\left(1-1 / 2 \omega^{2} L C\right) \text {. }
$$

When filling in typical numbers for coaxial cables, i.e., $300 \mathrm{nH} / \mathrm{m}$ and $100 \mathrm{pF} / \mathrm{m}$, and taking into account the $40 \mathrm{pF}$ input impedance of the DUT, we find that for a cable of $75 \mathrm{~cm}$ plus $4 \mathrm{~cm}$ connector the deviation should be around $560 \mu \mathrm{V} / \mathrm{V}$, which is $10 \%$ higher than the measured value. This means that the cable parameters (length or characteristic impedance or capacitance) are slightly overestimated or the measurement results are about $10 \%$ too low, which is still well within the uncertainty of $100 \mu \mathrm{V} / \mathrm{V}$.

When considering the voltage signal starting at the Josephson chip at position $x=0$ and propagating through the cable with full reflection at position $x=l$ by the high-ohmic input impedance of the meter under test, the voltage amplitude $V(x)$ along the cable relative to the voltage $V_{\text {out }}$ at the point of reflection can be written as

$$
V(x) / V_{\text {out }}=\left(\mathrm{e}^{\mathrm{j} \mathrm{k}(\mathrm{x}-\mathrm{l})}+\mathrm{e}^{-\mathrm{jk}(\mathrm{x}-\mathrm{l})}\right) / 2,
$$

where the wave number $k$ is equal to $k=2 \pi f / v, v$ being the speed of propagation of electromagnetic waves through the cable. At $x=0$ this is equal to $\left[1-2 \pi^{2} \cdot(f \cdot l / v)^{2}\right]$ for waves with wavelength $f / v$ much smaller than the length of the cable which is the case for the frequencies considered here. A more detailed calculation, similar to the one used in [12], shows that the effect of the $40 \mathrm{pF}$ input capacitance on the reflection of waves is similar to increasing the length of the cable plus connector by $38 \mathrm{~cm}$ to $117 \mathrm{~cm}$. Filling in the values of the frequency of $1 \mathrm{MHz}$ and the apparent cable length of $113 \mathrm{~cm}$, for the observed deviation of $510 \mu \mathrm{V} / \mathrm{V}$ we find a propagation speed of $77 \%$ of the speed of light in vacuum, which is about $15 \%$ higher than the values specified for coaxial cables. This again indicates that the (apparent) length of the cable is slightly overestimated or the measured values are too low.

Note that when using the usual expression $v=1 / \sqrt{ }(L C)$, the equation used in the lumped circuit approach is recovered and we see that the two approaches are equivalent. However, the reflected waves approach might be helpful in understanding some other observations and might lead to new mitigation methods as well.

\section{F. Digitizer connected}

Furthermore, we investigated the influence of connecting other cables and equipment to the ACJVS. Starting again from the original configuration with just the bias tee between the ACJVS and the TTS, we connected the digitizer with 1 meter of cable to the tee connector (see Fig. 2). Even though the length between ACJVS and TTS was the same, the observed deviation changed from $610 \mu \mathrm{V} / \mathrm{V}$ to $0.28 \%$ at $1 \mathrm{MHz}$. Note that the latter value is almost the same as the value indicated in Fig. 8 for an extra cable of 1 meter between ACJVS and TTS.

Disconnecting the digitizer but keeping the 1 meter cable did not change the measurement value. Apparently the input impedance of the digitizer, in specific its input capacitance, did not have a noticeable influence on the measurement. This indicates that the observed deviations are indeed caused by reflection of waves at the end of this connection cable rather than by a resonance which is dependent on the value of the capacitance connected in parallel.

\section{G. LF compensation circuit connected}

Apart from varying the configuration at the input of the TTS, we also investigated the influence of connecting equipment to the ACJVS input. Starting from the first configuration with direct connection again with only the bias tee between ACJVS and TTS, we generated $20 \mathrm{mV}$ with low frequency compensation signal from battery-operated homebuilt compensation electronics and compared the values at $500 \mathrm{kHz}$ (the maximum frequency supported by the compensation circuit). The result showed an increase of the deviation at $500 \mathrm{kHz}$ from $150 \mu \mathrm{V} / \mathrm{V}$ to about $540 \mu \mathrm{V} / \mathrm{V}$. When disconnecting the compensation electronics but keeping the extra cable used to connect the compensation, only a slight increase of a few $\mu \mathrm{V} / \mathrm{V}$ was observed.

When generating $50 \mathrm{mV}$ with the compensation electronics the deviation was as large as $0.25 \%$ at $500 \mathrm{kHz}$. Using a voltage source with series resistor replacing the home-built compensation electronics this deviation was reduced to the value at $20 \mathrm{mV}$ with only the cable connected. This suggests that the compensation electronics used in this configuration complicates the circuit; however, further investigation of the use of the electronics is outside the scope of this work.

\section{CONCLUSIONS}

In this paper we have shown the results from AC-DC measurements performed using a pulse-driven ACJVS with a small cryostat enabling voltage leads of about $70 \mathrm{~cm}$ only. The deviation at higher frequency, which is the parameter to be corrected, was an order of magnitude smaller than was observed before with a normal cryostat and considerably longer voltage leads, that is, at $1 \mathrm{MHz}$ we measured a smallest 
deviation of $510 \mu \mathrm{V} / \mathrm{V}$ as compared to almost $10000 \mu \mathrm{V} / \mathrm{V}$ (or $1 \%$ ) measured in a normal cryostat. With this result the correction to be made for the voltage leads is an order of magnitude smaller and, consequently, more reliable.

The observed square dependence on the signal frequency and cable length follows exactly the expected behavior. All results agree well with the calibrations performed in a micropot system. Furthermore, the influence of other cables and equipment was investigated. The measurement results can be quantitatively understood both in terms of a lumped circuit description and in terms of reflected waves.

\section{ACKNOWLEDGMENT}

We thank R. Behr for valuable discussions, Susanne Weimann for providing us with the TTS calibration data, and R. Wendisch, P. Duda and G. Muchow for technical assistance.

\section{REFERENCES}

[1] S. P. Benz and C. A. Hamilton, "A pulse-driven Programmable Josephson voltage standard,” Appl. Phys. Lett., vol. 68, pp. 3171-3173, May 1996

[2] E. Houtzager, S. P. Benz, and H. E. van den Brom, "Operating Margin for a Pulse-Driven Josephson Arbitrary Waveform Synthesizer Using a Ternary Bit-Stream Generator,” IEEE Trans. Instr. Meas., vol. 58, no. 2, pp. 775-780, April 2009

[3] Benz S P, Waltman S B, Fox A E, Dresselhaus P D, Rüfenacht A, Underwood J M, Howe L, Schwall R E and Burroughs C J, "1 V Josephson arbitrary waveform synthesizer", IEEE Trans. Appl. Supercond. 25, pp. 1300108, 2015

[4] Kieler O F, Behr R, Wendisch R, Bauer S, Palafox L and Kohlmann J "Towards a $1 \mathrm{~V}$ Josephson arbitrary waveform synthesizer" IEEE Trans. Appl. Supercond. 25, pp. 1400305, 2015

[5] B. Jeanneret, A. Rüfenacht, F. Overney, H. E. van den Brom and E. Houtzager, "High precision comparison between a programmable and a pulse-driven Josephson voltage standard", Metrologia 48, pp. 311-316, 2011

[6] Ralf Behr, Oliver Kieler, Jinni Lee, Stephan Bauer, Luis Palafox and Johannes Kohlmann , "Direct comparison of a $1 \mathrm{~V}$ Josephson arbitrary waveform synthesizer and an ac quantum voltmeter", Metrologia, Volume 52, Number 4, pp. 528-537, 2015

[7] Piotr S. Filipski, Helko E. van den Brom, and Ernest Houtzager, "International comparison of quantum AC voltage standards for frequencies up to $100 \mathrm{kHz}$," Measurement, Vol. 45, pp. $2218-2225$, 2012

[8] O. F. Kieler, R. Behr, D. Schleussner, L. Palafox, J. Kohlmann, "Precision Comparison of Sine Waveforms With Pulse-Driven Josephson Arrays", IEEE Trans. Appl. Supercond., Vol. 23, pp. , 2013

[9] T. E. Lipe, J. R. Kinard, Y. H. Tang, S. P. Benz, C. J. Burrroughs, and P. D. Dresselhaus, "Thermal voltage converter calibrations using a quantum AC standard," Metrologia, vol. 45, pp. 275-280, May 2008

[10] P. S. Filipski, M. Boecker, S. P. Benz and C. J. Burroughs, "Experimental determination of the voltage lead error in an ac Josephson voltage standard," IEEE Trans. Instrum. Meas., Vol. 60, pp. 2387 2392, 2011

[11] Helko E. van den Brom and Ernest Houtzager, "Voltage lead corrections for a pulse-driven ac Josephson voltage standard," Meas. Sci. Technol., Vol. 23, pp. 124007 - 124013, 2012

[12] Helko E. van den Brom, Dongsheng Zhao, and Ernest Houtzager, "Voltage lead errors in an AC Josephson voltage standard: explanation in terms of standing waves," CPEM Conf. Digest 2016

[13] Helko E. van den Brom, Oliver F.O. Kieler, Stephan Bauer, and Ernest Houtzager, "AC-DC Calibrations With a Pulse-driven AC Josephson Voltage Standard Operated in a Small Cryostat", CPEM Conf. Dig. 2016

[14] O. F. Kieler, D. Schleußner, J. Kohlmann, and R. Behr, "Josephson arbitrary waveform synthesizer for analysis of AC components," CPEM 2010 Conf. Digest, Daejeon, Korea, pp. 157-158, 2010
[15] H. E. van den Brom, E. Houtzager, B. E. R. Brinkmeier, and O. A. Chevtchenko, "Bipolar pulse-drive electronics for a Josephson arbitrary waveform synthesizer," IEEE Trans. Instrum. Meas., vol. 57, no 2, pp. 428-431, Feb. 2008.

[16] S.P. Benz, C.J. Burroughs, and P.D. Dresselhaus, "AC coupling technique for Josephson waveform synthesis", IEEE Trans. Appl. Supercond., Vol. 11, pp. 612-616, Mar. 2001

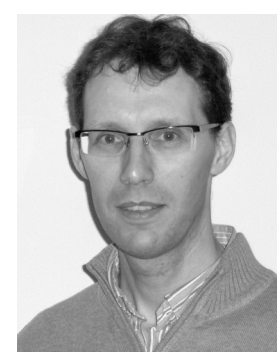

Helko E. van den Brom (M'14SM'15) was born in Utrecht, the Netherlands, in 1971. He received the M.Sc. degree in theoretical solid state physics from Utrecht University in 1995, and the Ph.D. degree in experimental solid state physics from Leiden University, the Netherlands, in 2000.

In 2000, he joined VSL, Delft, the Netherlands, where he started working on the development of Josephson and SET based electrical quantum standards. At present, as a principle scientist and project manager he is responsible for calibration and development of the national DC and low frequency electrical standards. His current research interests are in DC and AC Josephson voltage standards, very small DC currents, power quality, current transformers and sampling systems.

Dr. Van den Brom is a member of the Dutch Physical Society (NNV), technical assessor for the Dutch Accreditation Council (RvA), contact person for VSL in the technical committee of electricity and magnetism (TCEM) and the subcommittee of DC and quantum electrical measurements of the European Association of National Metrology Institutes (EURAMET). He was a recipient of the best Ph.D. paper award of the Dutch Journal of Physics (NTvN) in 2000.

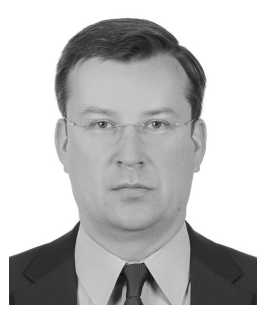

Oliver Kieler was born in Halberstadt, Germany, on November 11, 1969. He received the M.S. and Ph.D. degrees in physics from Otto-von-Guericke University of Magdeburg, Magdeburg, Germany, in 1995 and 1998, respectively. His thesis research focused on the development of extractor gauges for extreme high-vacuum applications.

In 1999, he transferred to the Otto-von-Guericke University, where he was involved in thin-film deposition and surface analysis techniques for the development of cold electron emitter-based monitor displays. Since 2000, he has been with the Physikalisch-Technische Bundesanstalt, Brunswick, Germany. In the Semiconductor Group, he worked on single-electron transport for the realization of a quantum-based current standard. He is currently working in the Quantum Voltage Standard Group, where he is focused on the design, fabrication, and measurement of Josephson junction arrays. Another field of research is the development of NanoSQUIDs. He was also a Visiting Guest Researcher at the National Institute of Standards and Technology (NIST, Boulder, Co, USA) for three months each in 2006 and 2009. 
Dr. Kieler was the recipient of a Fellowship from the State of Saxony-Anhalt from 1995 to 1998 to pursue the Ph.D. degree.

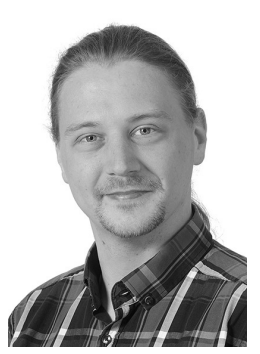

Stephan Bauer was born in Münster, Germany, on July 26, 1983. He received the Diplom and Ph.D. degrees in physics from Westfälische-Wilhelms University Münster, Germany, in 2009 and 2014, respectively. His thesis research focused on the energy calibration and stability monitoring of the direct neutrino mass experiment KATRIN.

In 2013 he joined the Josephson Voltage Group at Physikalisch-Technische Bundesanstalt. At present he is working on impedance bridges based on pulse driven Josephson Standards.

Dr. Bauer is member of the German Physical Society (DPG).

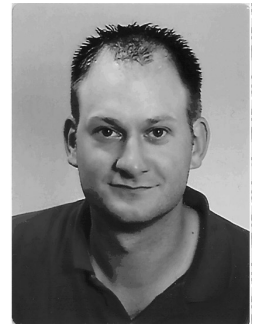

Ernest Houtzager (M'12-SM'14) was born in Hilversum, The Netherlands, in 1978. He received his B.Sc. degree in electronics from the Hanzehogeschool, Groningen, The Netherlands, in 2001.

After his studies he joined VSL (formally the NMi Van Swinden Laboratorium), Delft, The Netherlands, in 2001, where he specialized in high frequency and DC resistance measurements and became involved in the development of a quantum AC voltage standard. As from 2008, his main working area is in high voltage and low frequency power measurements. Other areas of expertise include quantum Hall and quantum voltage standards. 